\title{
Probing unitarity violation in the tail of the off-shell Higgs boson in $V_{L} V_{L}$ mode
}

\author{
Seung J. Lee, ${ }^{1,2, *}$ Myeonghun Park, ${ }^{3,4, \dagger}$ and Zhuoni Qian ${ }^{4, \$}$ \\ ${ }^{1}$ Department of Physics, Korea University, Seoul 136-713, Korea \\ ${ }^{2}$ School of Physics, Korea Institute for Advanced Study, Seoul 130-722, Korea \\ ${ }^{3}$ Seoul National University of Science and Technology, Seoul 01811, Korea \\ ${ }^{4}$ Center for Theoretical Physics of the Universe, Institute for Basic Science (IBS), Daejeon 34051, Korea
}

(Received 14 December 2018; published 16 July 2019)

\begin{abstract}
Off-shell Higgs bosons at the high mass tail may shed light on the underlying mechanism of the electroweak symmetry breaking. In the Standard Model, there is an exact cancellation of the logarithmic divergence between the box and Higgs-mediated triangle diagrams due to unitarity, such that the $g g \rightarrow$ $Z Z(W W)$ process is left with a negligible $V_{L} V_{L}$ longitudinal mode contribution in the tail region of the offshell Higgs boson. We propose to test the unitarity of the Standard Model by probing this behavior, as new physics violating this cancellation at certain tail region can be probed by a precision study of the $V_{L} V_{L}$ mode. To this end, we propose to utilize the information in angular observables to maximize the sensitivity of the $V_{L} V_{L}$ final states in the high mass tail region.
\end{abstract}

DOI: 10.1103/PhysRevD.100.011702

\section{INTRODUCTION}

With the discovery of the Higgs boson at the LHC [1,2], the Standard Model (SM) seems to be the true low energy effective theory (EFT), with the Higgs mechanism accounting for electroweak symmetry breaking (EWSB). Since so far there is no hint of new physics (NP) at the LHC, one of the biggest questions that will remain crucial and insightful is "how well do we know whether it is indeed the SM Higgs that restores unitarity, which is otherwise violated without it." A well-studied test is by looking at the $W W / Z Z$ boson scattering processes at a high energy scale to check that unitarity is indeed conserved. Less known is to test unitarity by looking at the longitudinal modes towards the off-shell region of the Higgs mass distribution, as the logarithmic divergence from the box and Higgs-mediated triangle diagrams in this mode exactly cancel each other in the SM. For this reason, the off-shell Higgs boson deserves a careful study for possible unitarity violation behavior due to deviation from the theory, and an optimal place to start would be the $h^{*} \rightarrow Z Z \rightarrow 4 \ell$ decay channel given its high precision [3-5]. This will be relevant for the ongoing LHC program and at the High-Luminosity LHC (HL-LHC),

\footnotetext{
sjjlee@korea.edu

parc.seoutech@seoutech.ac.kr

zhuoniq@ibs.re.kr
}

Published by the American Physical Society under the terms of the Creative Commons Attribution 4.0 International license. Further distribution of this work must maintain attribution to the author(s) and the published article's title, journal citation, and DOI. Funded by SCOAP. where a large amount of data is accumulated; thus, the LHC becomes a "Higgs factory" at hand and a high precision machine in the near future. Then, we can start our next journey by improving the sensitivity on testing the SM.

The relevance of the longitudinal mode at the high mass scale is expected by the Goldstone boson equivalence theorem, and it is thus natural to consider tagging the longitudinal polarization mode for improving our understanding of the Higgs sector. In fact, the importance of probing the longitudinal component of the vector bosons have long been discussed [6].

Depending on the different polarization combinations, the vector boson on-shell pair production channel could be categorized into TT (transverse-transverse), TL (transverselongitudinal), and LL (longitudinal-longitudinal) modes. In the SM, $Z$ boson pair production is mostly from the $q \bar{q} \rightarrow$ $Z Z$ process, which is dominated by the TT mode. For $g g \rightarrow Z Z$, the destructive interference between the box and Higgs-mediated triangle loop diagrams leaves the total cross section dominated by the TT mode. To understand the cancellation in the LL mode, we note that the contribution from the box diagram with massive quarks in the loop is dominated by the axial-axial current which scales as $C_{A}^{2}\left(\sqrt{\hat{s}} / m_{Z}\right)^{2}$, and the corresponding amplitude in the $\sqrt{\hat{s}} \gg m_{t}$ limit goes [7]

$$
\mathcal{A}_{\square} \rightarrow-8 C_{A}^{2} \frac{m_{t}^{2}}{\hat{s}}\left(\frac{\hat{s}}{m_{Z}^{2}}\right) \log ^{2}\left(\frac{\hat{s}}{m_{t}^{2}}\right)=-\frac{m_{t}^{2}}{2 m_{Z}^{2}} \log ^{2}\left(\frac{\hat{s}}{m_{t}^{2}}\right) .
$$

At high energy, this amplitude violates unitarity, and so is the amplitude from the Higgs-mediated triangle diagram, 


$$
\mathcal{A}_{\triangle} \rightarrow \frac{m_{t}^{2}}{\hat{s}} \frac{1}{2} \log ^{2}\left(\frac{m_{t}^{2}}{\hat{s}}\right)\left(\frac{\sqrt{\hat{s}}}{m_{Z}}\right)^{2}=\frac{m_{t}^{2}}{2 m_{Z}^{2}} \log ^{2}\left(\frac{\hat{s}}{m_{t}^{2}}\right) .
$$

The log-divergent terms from these two contributions cancel exactly, and unitarity is restored. However, this cancellation may not hold in the presence of NP. For example, a modification to the $g g \rightarrow V_{L} V_{L}$ amplitude at a high scale can be established from 1) a change in the Higgs propagator 2) the introduction of a new propagator 3) a variation in $h V_{L} V_{L}$ form factor. These cases can be portrayed by simplified models. A Higgs portal light scalar loop-corrects the Higgs self energy and modifies the Higgs propagator, correspondingly. A heavy Higgs-like scalar with a broad width contributes to $g g \rightarrow V_{L} V_{L}$ as an additional propagator. Finally, in the case of the quantum critical Higgs $(\mathrm{QCH})$ model, where the Higgs arises as a bound state of a conformal field theory with a gapped continuum above a certain scale, unitarity is restored at a much higher scale [8]. We demonstrate that tagging the LL mode in the tail region provides a sensitive probe to all these scenarios, which cannot be captured by a simple EFT analysis with higher dimensional operators. To study the deviation from a simple energy-growing behavior of a higher dimensional EFT operator, we consider a dimension8 operator, the lowest order that affects the $q \bar{q} \rightarrow Z Z$ process and grows with $\hat{s}^{2} / \Lambda^{4}$ [9],

$$
O^{(8)}=\frac{c_{8}}{\Lambda^{4}}\left(i \bar{\psi} \bar{\gamma} \gamma^{\{\mu} \partial^{\nu\}} \psi+\text { H.c. }\right) D_{\mu} H^{\dagger} D_{\nu} H .
$$

We do not consider operators that affect the $g g H$ coupling such as the dimension six operator, $|\Phi|^{2} G_{\mu \nu} G^{\mu \nu}$, given that such gg-initiated dimension-6 Higgs effective operators are well studied in the literature, and our focus is certainly not on generic EFT operator searches.

Experimental searches in the high mass tail of the $Z Z$ final states exist for specific NP cases, for example, heavy resonance searches [10,11], and indirect bounds on a deviation of the Higgs total width [12-15]. For a general search to find hints of NP in the Higgs sector, however, we cannot rely on those dedicated analyses which require model-specific information. We present a model-independent and effective method to cover generic NP scenarios modifying the Higgs sector.

\section{NEW PHYSICS WITHIN THE LHC SCALE}

\section{A. Higgs portal light scalar}

As studied in [16], a Higgs portal light scalar with a mass of $m_{S}>m_{h} / 2$, which evades constraints from Higgs invisible decay searches, would contribute through loop effects to the Higgs self energy and modify the $m_{Z Z}$ distribution at a high energy scale. Such a scalar that couples only through the Higgs boson without mixing is otherwise poorly constrained except through the precision measurements of the Higgs inclusive production at a future lepton collider [17-19]. To study, we write a simplified Lagrangian of a SM plus a complex scalar in the form,

$$
\mathcal{L} \ni \mathcal{L}_{\mathrm{SM}}+\partial_{\mu} S \partial^{\mu} S^{*}-\mu^{2}|S|^{2}-\kappa|S|^{2}|\Phi|^{2} .
$$

At next leading order (NLO), the scalar $S$ modifies the Higgs propagator through one loop, and the renormalized self-energy becomes

$$
\hat{\Sigma}_{h}(s)=\Sigma_{h}\left(p^{2}\right)-\delta \mu_{h}^{2}+\left(p^{2}-\mu_{h}^{2}\right) \delta Z_{h} .
$$

$\mu_{h}^{2}$ is the squared complex mass defined as $\mu_{h}^{2}=m_{h}^{2}-$ $i m_{h} \Gamma_{h} . \delta Z_{h}$ and $\delta \mu_{h}^{2}$ are the wave function and mass renormalization of the Higgs field, respectively. In the on-shell scheme, they are defined as

$$
\delta \mu_{h}^{2}=\Sigma_{h}\left(\mu_{h}^{2}\right), \quad \delta Z_{h}=-\frac{d \Sigma_{h}}{d p^{2}}\left(\mu_{h}^{2}\right) .
$$

Exact NLO precision is calculated by truncating to an order $\mathcal{O}\left[\left(\hat{\Sigma}_{h}\left(p^{2}\right)\right)^{2}\right]$ at the squared amplitude level, with the modified propagator,

$$
\text { Propagator }^{\mathrm{NLO}} \ni \frac{i}{p^{2}-\mu_{h}^{2}}\left(1-\frac{\hat{\Sigma}_{h}\left(p^{2}\right)}{p^{2}-\mu_{h}^{2}}\right) .
$$

This deviation at high energy becomes apparent in the LL mode as expected. We set the benchmark point with $\kappa=9$ and $m_{S}=80 \mathrm{GeV}$, where $\kappa$ is set to be the largest allowed value by current experimental searches $[10,20]$. Note that perturbative constraints from quantum corrections require $\kappa$ to be smaller than around 5-6 as discussed in Ref. [21] in this simplified model. However, we consider a larger value of $\kappa$ to cover a broad range of probable NP beyond a simplified version.

\section{B. Broad-width heavy scalar}

Another example is a heavy scalar $S$ decaying to $Z Z$ through its mixing with the SM scalar. Here, we take a representative example of an additional real scalar as

$$
\mathcal{L} \ni \mathcal{L}_{\mathrm{SM}}+\frac{1}{2} \partial_{\mu} S \partial^{\mu} S-\frac{1}{2} m_{S} S^{2}-\mu_{S} S|\Phi|^{2} .
$$

After EWSB, there is mixing between the $S$ and the Higgs boson $h$ with the mixing angle $\tan \theta=\frac{\mu_{S} v}{\sqrt{\left(\mu_{S} v\right)^{2}+\left(m_{S}^{2}-m_{h}^{2}\right)^{2}}}$. Here, $v$ is the SM vacuum expectation value. In the limit of small mixing and $m_{S}^{2} \gg m_{h}^{2}, \sin \theta \sim \mu_{S} v / m_{S}^{2}$ [22]. We take the scalar mass at $M_{S}=700 \mathrm{GeV}$ and $\sin \theta=0.4$ as still allowed by current Higgs data [23] and assume a relatively broad width $\Gamma_{S}=140 \mathrm{GeV}$, which manifests large interference. This corresponds to $\mu_{S} \sim 4.6 v$ and the decay width of the heavy scalar to $\mathrm{SM}$ around $30 \mathrm{GeV}$, dominated by 
$S \rightarrow h h$. The broad width we assume could arise from the real scalar $S$ decaying to a hidden sector, which could eventually decay back to soft SM final states. This case is also constrained by monojet searches [24].

\section{Quantum critical Higgs boson}

Models of QCH [8,25-27] typically predict a higher scale continuum which modifies the Higgs off-shell region. The natural version of QCH can be built by implementing the original model in the warped extra dimension with a linear dilaton setup, as in the continuum naturalness framework [28], except that the Higgs boson here would be represented by a generic bulk scalar field, instead of the fifth component of a bulk gauge field corresponding to a 4D pseudo-Nambu-Goldstone boson. In general, the Higgs couplings to the other SM particles could depend on the details of the UV theory, conformal symmetry breaking, and the scale. We consider a minimal scenario where the propagator of the physical Higgs field and the $h Z Z$ coupling are modified as follows:

$$
\begin{aligned}
G_{h}(p) & =-\frac{i Z_{h}}{\left(\mu^{2}-p^{2}-i \epsilon\right)^{2-\Delta}-\left(\mu^{2}-m_{h}^{2}\right)^{2-\Delta}}, \\
g_{h Z Z} & =-\frac{\left(\mu^{2}\right)^{2-\Delta}-\left(\mu^{2}-p^{2}\right)^{2-\Delta}}{\hat{s}} g_{h Z Z}^{S M} .
\end{aligned}
$$

The nonstandard $h Z Z$ form factor arises from the gauge invariant form of the Higgs two-point function. The continuum scale $\mu$ and the anomalous dimension $\Delta$ are the two new parameters in this simplified case. We chose $\mu=600 \mathrm{GeV}$ and $\Delta=1.6$ as a benchmark point, which is still allowed by the current experimental search in the high mass tail of the $Z Z$ final states [10].

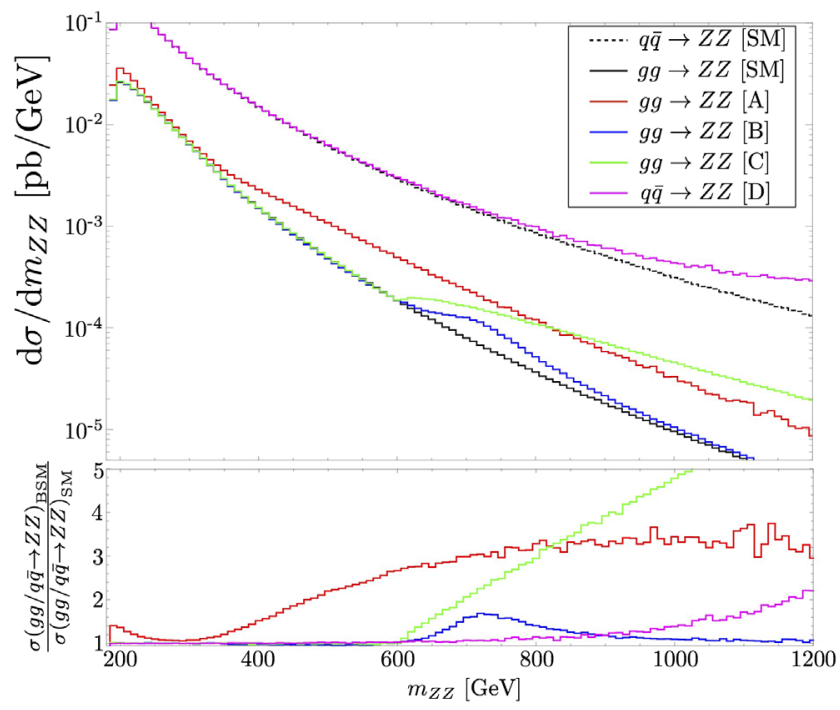

\section{ANALYSIS}

Focusing on the polarization composition of the NP cases from Fig. 1, we see that the deviation of the BSM compared to the SM in the total rate (left plot) is mainly from the deviation in the LL mode (right plot). Given this physical feature, we enhance the experimental sensitivity on generic Higgs sector new physics, that hides at a high energy scale.

We use MADGRAPH5_AMC@NLO [29] to generate $g g(q \bar{q}) \rightarrow Z Z \rightarrow e^{-} e^{+} \mu^{-} \mu^{+}$events at QCD leading order and rescale with a $\mathrm{k}$ factor of 1.8 (1.5) for the $g g(q \bar{q})$ initiated processes, respectively $[30,31]$. For NP examples, we generate $g g \rightarrow Z Z \rightarrow e^{-} e^{+} \mu^{-} \mu^{+}$events for the light scalar case (case A), heavy Higgs case (case B), and QCH model (case C) with model parameters given in Sec. II. Generator level cuts are applied as $p_{T \ell}>10 \mathrm{GeV}$, $\left|\eta_{\ell}\right|<2.5, m_{\ell^{-} \ell^{+}}>50 \mathrm{GeV}$, and $m_{4 \ell}>560 \mathrm{GeV}$. After detector simulation with DeLPHES [32], we further require in the final state a pair of electrons and muons with baseline selections,

$$
80<m_{l l}<100 \mathrm{GeV}, \quad m_{4 l}>600 \mathrm{GeV} .
$$

To develop model-independent cuts, we simulate a generic NP signal with $g g \rightarrow h^{*} \rightarrow 4 \ell$ process which is reweighted by a modified propagator with $m_{h}=m_{4 \ell}$. By performing the reweighting, we remove the dependence on the scale of a specific NP. The backgrounds are the SM $q \bar{q} \rightarrow Z Z$ and $g g \rightarrow Z Z$ processes, dominated by the TT mode in the high mass scale.

One variable we find useful is $\cos \theta^{*}$. It's the $\operatorname{cosine}$ of the angle between one final state $Z$ that is reconstructed

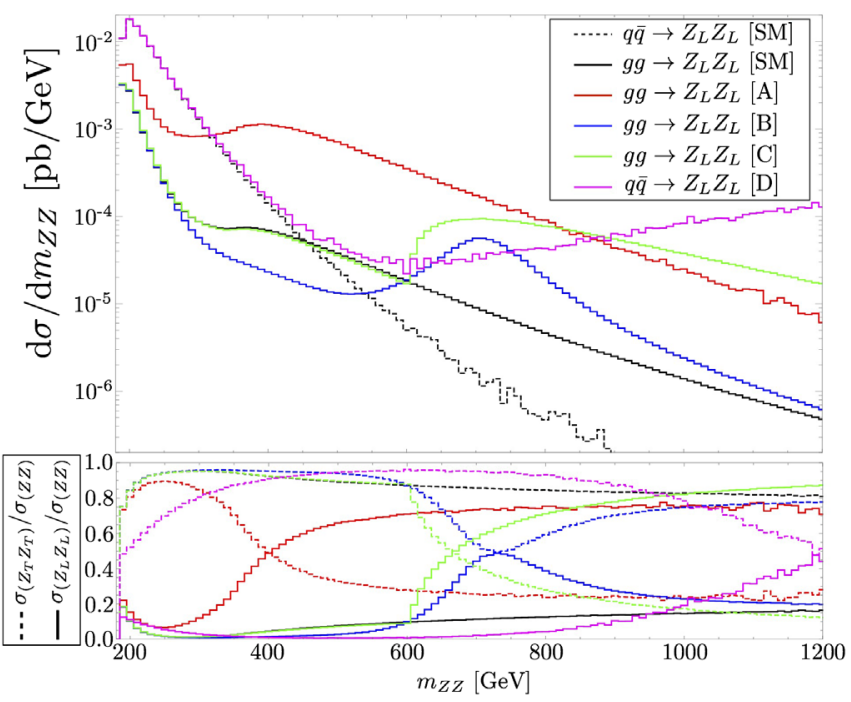

FIG. 1. (Left top) The differential cross sections of $q \bar{q} \rightarrow Z Z, g g \rightarrow Z Z$ for relevant processes, and the ratio (left bottom) between the physics beyond the Standard Model (BSM) cases A, B, C, D, and the SM backgrounds, where D represents the case with a dimension- 8 effective operator. (Right top) The differential cross section in the LL polarization mode for relevant processes, and the ratio (right bottom) of LL and TT mode components compared to the total rate for $g g(q \bar{q}) \rightarrow Z Z$ processes. 
TABLE I. Achievable significance for the NP cases at $3 \mathrm{ab}^{-1}$ HL-LHC with benchmark points. Baseline selections are defined in Eq. (8) and generic angle cuts (gen.) in Eq. (10). Optimal cuts (opt.) are described in the text of Sec. III.

\begin{tabular}{lccc}
\hline \hline Significance $\sigma$ & Case A & Case B & Case C \\
\hline Baseline selections & 1.40 & 0.441 & 3.28 \\
$m_{4 \ell}$ cuts (gen/opt) & $0.826 / 1.40$ & $0.233 / 0.716$ & $4.19 / 4.20$ \\
Angle cuts (gen/opt) & $2.02 / 2.10$ & $0.727 / 0.732$ & $5.02 / 5.12$ \\
KD cut (gen/opt) & $2.14 / 2.24$ & $0.850 / 0.857$ & $6.56 / 6.66$ \\
BDT (gen/opt) & $2.16 / 2.53$ & $0.812 / 1.20$ & $6.19 / 7.12$ \\
\hline \hline
\end{tabular}

from $\mu^{-} \mu^{+}$pair in our analysis, with respect to the beam line in the center of mass frame. The $\cos \theta^{*}$ distribution for the signal is flat, as expected from an s-channel scalar mediator. For the major background $q \bar{q} \rightarrow Z Z$, the distribution approaches $\frac{1+\cos ^{2} \theta^{*}}{1-\cos ^{2} \theta^{*}}$ in the limit of $\sqrt{\hat{s}} \gg m_{Z}$, which is a good approximation at the investigated energy scale. We also apply a cut on the angle $\cos \theta_{1}$ between the direction of the leading- $p_{T} Z$ and the negatively charged fermion from its decay in its rest frame. The $\cos \theta_{i}$ distribution is determined by the $Z_{i}$ polarization as follows:

$$
\frac{\mathrm{d} \sigma}{\mathrm{d} \cos \theta_{i}} \propto \begin{cases}1-\cos ^{2} \theta_{i} & \left(\text { when } Z_{i}=Z_{L}\right) \\ 1+\cos ^{2} \theta_{i} & \left(\text { when } Z_{i}=Z_{T}\right)\end{cases}
$$

To capture generic NP signals, we choose the cuts on angular variables at the maximum of $S / \sqrt{B}$, where signal events are from a generic NP signal of $g g \rightarrow h^{*} \rightarrow 4 \ell$,

$$
\left|\cos \theta^{*}\right|<0.7, \quad\left|\cos \theta_{1}\right|<0.68
$$

To further utilize the multidimensional information in the final state, we perform a general KD-variable cut [33], where

$$
\mathrm{KD}=\ln \left(\frac{f_{g}\left(x_{1}\right) f_{g}\left(x_{2}\right)\left|\mathcal{M}\left(g g \rightarrow h^{*} \rightarrow 4 \ell\right)\right|^{2}}{\sum f_{q}\left(x_{1}\right) f_{\bar{q}}\left(x_{2}\right)|\mathcal{M}(q \bar{q} \rightarrow 4 \ell)|^{2}}\right),
$$

which is the ratio of the squared amplitudes between our generic signal and dominant $q \bar{q}$ background processes, weighted with the respective PDFs. As in our generic signal events, we set $m_{h}=m_{4 \ell}$ in the matrix element calculation. Additionally, we perform a boosted decision tree (BDT) analysis to combine six variables: $m_{4 \ell}, \cos \theta^{*}, \cos \theta_{1}$, $\cos \theta_{2}, \Delta \phi$, and KD. $\Delta \phi$ is the angle between the two decay planes of the $Z$ bosons. The BDT cut is trained on the generic signal against the dominant $q \bar{q}$ background and applied to all processes. To check how much these generic cuts are optimized for individual NP cases, we perform a case-by-case optimization of cuts (BDT training) with different methods: $m_{4 \ell}$, angular, KD, and BDT. In Table I, we list the significance achieved at the baseline selections, angular cuts, KD cut, and BDT cut, respectively. We include a $5 \%$ systematic error from the dominant background $q \bar{q} \rightarrow Z Z$ process [11]. Systematic errors for the $g g$ initiated processes are neglected, since we are taking the difference between the NP and SM. Both the generic (gen) cuts and the case-by-case optimized (opt) cuts are shown. To visualize performances, we plot receiver operating characteristic-curve in Fig. 2, which shows the discrimination power of a binary classifier based on a given cut for a specific NP. As we observe, angular cuts which utilize the information of Z-boson polarization are powerful enough as we do not see much further enhancement from KD or BDT analyses.

\section{DIMENSION-8 EFT OPERATOR}

The dimension-8 operator gives rise to an energy dependent $q \bar{q} Z_{L} Z_{L}$ 4-point interaction. The relevant term for our $p p \rightarrow Z Z$ process, constrained by unitarity/analyticity [34,35] reads [9] case A

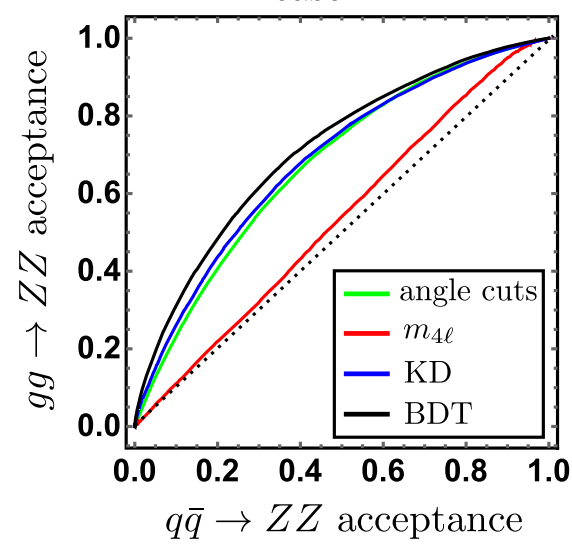

case B

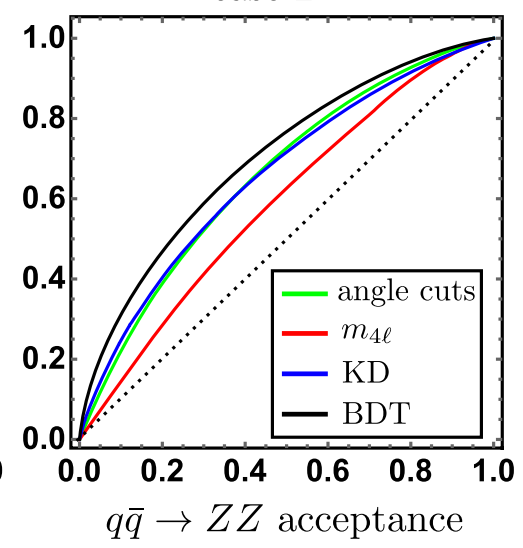

case $\mathrm{C}$

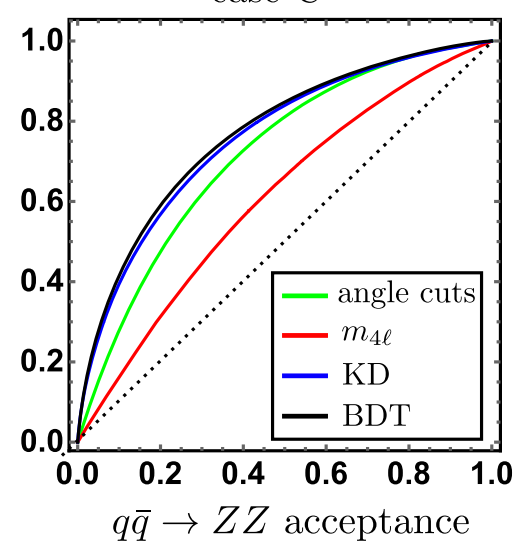

FIG. 2. Receiver operating characteristic-curve shows the acceptance rate of $g g$-initiated processes against the SM $q \bar{q}$-initiated one. Conventional $m_{4 \ell}$-based kinematic cuts are not sensitive compared to cuts based on polarization information. Angular cuts are optimized enough so we do not expect much further enhancement from the KD and BDT procedure. 


$$
-\frac{c_{8}}{\Lambda^{4}} \frac{i g_{Z}^{2} v^{2}}{32}\left(\bar{\psi}_{q} \gamma^{\mu} \partial^{\nu} \psi_{q}+\bar{\psi}_{q} \gamma^{\nu} \partial^{\mu} \psi_{q}+\text { H.c. }\right) Z_{\mu} Z_{\nu}
$$

Note that this dimension- 8 operator can arise from the tree level diagram with a spin-2 mediator, as well as nonlinear SUSY models [35] when other dimension-8 operators are suppressed or forbidden by an underlying symmetry. With the benchmark point $\Lambda=1.26 \mathrm{TeV}, c_{8}=4 \pi$; the deviation mainly shows up in the LL mode as presented in Fig. 1. The enhancement of the LL mode at a high invariant mass tail, with $\hat{s}^{2}$ at the amplitude level, is due to the EFT operator, as the interference with the SM is highly suppressed.

This scenario with adding a dimension- 8 EFT operator is however different from the scenario A,B,C. Specifically, the $\cos \theta^{*}$ distribution is proportional to $\sin ^{2}\left(2 \theta^{*}\right)$ with a distinct dual-peak structure. An angular cut of $\left|\cos \theta_{1}\right|<$ 0.68 to favor the LL mode finds a 7\% improvement on the sensitivity. We then adopt a BDT analysis over variables $\theta^{*}, \theta_{1}, \theta_{2}, \phi, m_{4 \ell}$, and found the $m_{4 \ell}$ variable most effective in discrimination, followed by $\cos \theta^{*}$ and $\cos \theta_{i}$. This is expected due to the sensitive energy dependence of the dimension- 8 operator. We find that with $100 \mathrm{fb}^{-1}$ data of the $13 \mathrm{TeV}$ LHC, a cut of $\cos \theta_{1}<0.68$ constrains this operator to $\Lambda>1.3 \mathrm{TeV}$ at $1 \sigma$, and BDT analysis improves the bound to $1.5 \mathrm{TeV}$.

As studied in Ref. [9], there are in general $Z_{T} Z_{T}$ channels that get enhanced as well. The reach to these TT mode enhancing operators is comparable to the LL mode at around $\Lambda>1.3 \mathrm{TeV}$, due to the interference with a larger SM TT mode but weaker center-of-mass energy enhancement.

\section{SUMMARY AND DISCUSSION}

In this paper, we study the high mass tail region of the $p p \rightarrow h^{*} \rightarrow 4 \ell$ channel, which provides a sensitive test ground for the unitarity of the SM due to the presence of the Higgs boson. We point out that since the LL mode is suppressed in the SM due to unitarity, a precision study to look for a deviation in the longitudinal mode of $Z$ bosons provides an important window to test the SM. For this, we show that the sensitivity for deviation from the SM can be improved by utilizing angular cuts to enhance the LL mode. To illustrate our point, we examine the $g g \rightarrow Z Z$ channel with three simplified models of NP scenarios that modify the Higgs sector. Despite having different energy dependence from each scenario, we found that the angular cuts, favoring the central and LL mode, improves the significance by $40 \%-70 \%$ across all scenarios. A generic BDT analysis additionally improves all significance by about 10\%-20\%. This implies that we will be able to test the SM with much more accuracy at the HL-LHC, by looking for a deviation in the $Z_{L} Z_{L}$ mode at high mass tail region.

As a bonus of this study, we also examine the effects from a simple energy growing behavior of an EFT operator. For this purpose, we choose to examine a $q \bar{q}$-initiated dimension-8 EFT operator which could arise to importance over the dimension- 6 ones favored by certain types of NP models [9].

Note that our method is applicable to any diboson and Higgs associated production channels. A combined search can be extended straightforwardly from our study, and accumulatively further increase our knowledge of "how well we know that it is indeed the SM Higgs."

\section{ACKNOWLEDGMENTS}

The authors are grateful for conversations with Tao Han. This work was supported by the National Research Foundation of Korea (NRF) grant funded by the Korea government (MEST) (Grant No. NRF2015R1A2A1A15052408). S. L. was supported by Samsung Science and Technology Foundation under Project No. SSTF-BA1601-07. M.P. is supported by Basic Science Research Program through the National Research Foundation of Korea Research Grant No. NRF2018R1C1B6006572. M. P. and Z. Q. are supported by IBS under the Project No. IBS-R018-D1.
[1] G. Aad et al. (ATLAS Collaboration), Phys. Lett. B 716, 1 (2012).

[2] S. Chatrchyan et al. (CMS Collaboration), Phys. Lett. B 716, 30 (2012).

[3] C. Englert, Y. Soreq, and M. Spannowsky, J. High Energy Phys. 05 (2015) 145.

[4] A. Azatov, C. Grojean, A. Paul, and E. Salvioni, Zh. Eksp. Teor. Fiz. 147, 410 (2015) [J. Exp. Theor. Phys. 120, 354 (2015)].

[5] D. Goncalves, T. Han, and S. Mukhopadhyay, Phys. Rev. D 98, 015023 (2018).
[6] T. Han, D. Krohn, L. T. Wang, and W. Zhu, J. High Energy Phys. 03 (2010) 082.

[7] U. Baur, E. W. N. Glover, and J. J. van der Bij, Nucl. Phys. B318, 106 (1989).

[8] B. Bellazzini, C. Csâki, J. Hubisz, S. J. Lee, J. Serra, and J. Terning, Phys. Rev. X 6, 041050 (2016).

[9] B. Bellazzini and F. Riva, Phys. Rev. D 98, 095021 (2018).

[10] A. M. Sirunyan et al. (CMS Collaboration), J. High Energy Phys. 06 (2018) 127.

[11] M. Aaboud et al. (ATLAS Collaboration), Eur. Phys. J. C 78, 293 (2018). 
[12] N. Kauer and G. Passarino, J. High Energy Phys. 08 (2012) 116.

[13] F. Caola and K. Melnikov, Phys. Rev. D 88, 054024 (2013).

[14] V. Khachatryan et al. (CMS Collaboration), Phys. Lett. B 736, 64 (2014).

[15] M. Aaboud et al. (ATLAS Collaboration), Phys. Lett. B 786, 223 (2018).

[16] D. Goncalves, T. Han, and S. Mukhopadhyay, Phys. Rev. Lett. 120, 111801 (2018); 121, 079902(E) (2018).

[17] D. M. Asner et al., arXiv:1310.0763.

[18] M. L. Mangano, https://dx.doi.org/10.1142/9789813238053_ 0017.

[19] F. An et al., Chin. Phys. C 43, 043002 (2019).

[20] S. K. Kang, Z. Qian, J. Song, and Y. W. Yoon, Phys. Rev. D 98, 095025 (2018).

[21] D. Curtin, P. Meade, and C. T. Yu, J. High Energy Phys. 11 (2014) 127.

[22] L. Di Luzio, J. F. Kamenik, and M. Nardecchia, Eur. Phys. J. C 77, 30 (2017).

[23] J. Tao (CMS Collaboration), Nucl. Part. Phys. Proc. 300302, 53 (2018).

[24] A. Falkowski, O. Slone, and T. Volansky, J. High Energy Phys. 02 (2016) 152.
[25] D. Stancato and J. Terning, J. High Energy Phys. 11 (2009) 101.

[26] A. Falkowski and M. Perez-Victoria, J. High Energy Phys. 12 (2008) 107.

[27] A. Falkowski and M. Perez-Victoria, Phys. Rev. D 79, 035005 (2009).

[28] C. Csáki, G. Lee, S. J. Lee, S. Lombardo, and O. Telem, J. High Energy Phys. 03 (2019) 142.

[29] J. Alwall, R. Frederix, S. Frixione, V. Hirschi, F. Maltoni, O. Mattelaer, H.-S. Shao, T. Stelzer, P. Torrielli, and M. Zaro, J. High Energy Phys. 07 (2014) 079.

[30] F. Caola, K. Melnikov, R. Rontsch, and L. Tancredi, Phys. Rev. D 92, 094028 (2015).

[31] F. Cascioli, T. Gehrmann, M. Grazzini, S. Kallweit, P. Maierhöfer, A. von Manteuffel, S. Pozzorini, D. Rathlev, L. Tancredi, and E. Weihs, Phys. Lett. B 735, 311 (2014).

[32] J. de Favereau, C. Delaere, P. Demin, A. Giammanco, V. Lemaître, A. Mertens, and M. Selvaggi (DELPHES 3 Collaboration), J. High Energy Phys. 02 (2014) 057.

[33] P. Avery et al., Phys. Rev. D 87, 055006 (2013).

[34] A. Adams, N. Arkani-Hamed, S. Dubovsky, A. Nicolis, and R. Rattazzi, J. High Energy Phys. 10 (2006) 014.

[35] B. Bellazzini, J. High Energy Phys. 02 (2017) 034. 